\title{
COVID-19 Cases Spread Through the K350 Train — Jilin and Heilongjiang Provinces, China, January 2021
}

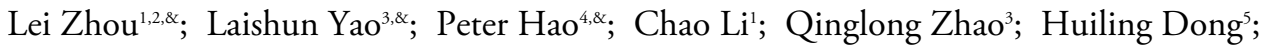 \\ Jinhua Dou'; Shengnan Wang;; George F. Gao ${ }^{4}$; Qun Li ${ }^{1, *}$; Biao Huang ${ }^{3, *}$
}

As of $16: 00$ on January 13,2021, 95 positive cases of coronavirus disease 2019 (COVID-19) were reported in Suihua City of Heilongjiang Province and an additional 35 cases have been linked across 7 cities in 3 provinces: Qiqihar City (7 cases), Mudanjiang City (4 cases), Harbin City (3 cases), and Yichun City (1 case) of Heilongjiang Province; Changchun City (9 cases) and Tonghua City (6 cases) of Jilin Province; and Weihai City of Shandong Province (1 case).

On January 10, 2021, the first asymptomatic infections in Changchun City of Jilin Province were found in couple recently traveling from Suihua City on January 5 (Suihua A and B; 58-year-old male and 57year-old female, respectively). After hearing of the outbreak in Suihua City, the couple voluntarily sought testing at the Second Hospital of Jilin University and returned positive COVID-19 tests. The initial investigation of the couple patients indicated that they had traveled from Suihua City to Changchun by the K350 Train on January 5. This launched an epidemiological investigation of close contacts and for other passengers of the K350 train on January 5.

\section{INVESTIGATION AND FINDINGS}

On January 11, 2021, close contact tracing found that the son of Suihua A and B had tested positive for COVID-19. On January 12, nucleic acid screening of the K350 train found 3 more asymptomatic infections in passengers: 2 residing in Changchun City (Changchun A and B; 53-year-old male and 48-yearold female, respectively) and 1 residing in Tonghua City (Tonghua A; 44-year-old male). These 5 patients all rode in the same passenger car of the K350 train and were in relatively close proximity as shown in Figure 1. Another 4 asymptomatic infections were detected in close contacts of Tonghua A, which resulted in a total of 10 infections linked to K350 in Jilin Province as of January 13. Table 1 shows a partial timetable of the times Suihua A and B, Changchun A and $\mathrm{B}$, and Tonghua $\mathrm{A}$ were found to be aboard K350 together.

Suihua A and B were likely infected while visiting relatives in Suihua and began experiencing symptoms on January 3, which subsided by the time they were tested on January 10. The couple boarded the K2209 train on December 28, 2020 and arrived in Suileng County of Suihua at 05:00 on December 29. They attended a large gathering for dinner involving approximately 20 people including the father of the index patient of Wangkui County of Suihua who became ill on January 3, 2021 and was diagnosed on January 10. On December 30, 2020, Suihua A and B attended a wedding involving approximately 190 people and then had dinner at Suihua A's uncle's home, at which Suihua A's younger brother (Qiqihar City's index patient; experienced symptoms from January 6 to 9 and was diagnosed on January 12) and younger sister (Yichun City's index patient; voluntarily sought testing and was diagnosed on January 11). On January 3, Suihua A and B began experiencing symptoms of dizziness and drowsiness; they made a warm ginger drink but did not seek medical care.

Suihua A and B boarded K350 at roughly 14:30 from Suihua Station and alighted at 20:00 at Changchun Station. Changchun A and B boarded K350 at roughly 16:30 from Harbin West Station of Heilongjiang Province and alighted at 20:00 at Changchun Station. Tonghua A boarded K350 at roughly 11:20 from Nancha Station in Heilongjiang Province and alighted at 16:30 at Harbin West Station. However, Tonghua A took additional trains after completing his journey from January 7 to 9 including Z174, K1383, and D124. Further investigations are being conducted.

Tonghua A conducted a work training seminar from January 10 to 11,2021 in Tonghua City. After Tonghua A was confirmed to be COVID-19 positive, 4 asymptomatic infections were found among attendees of the training seminar. 


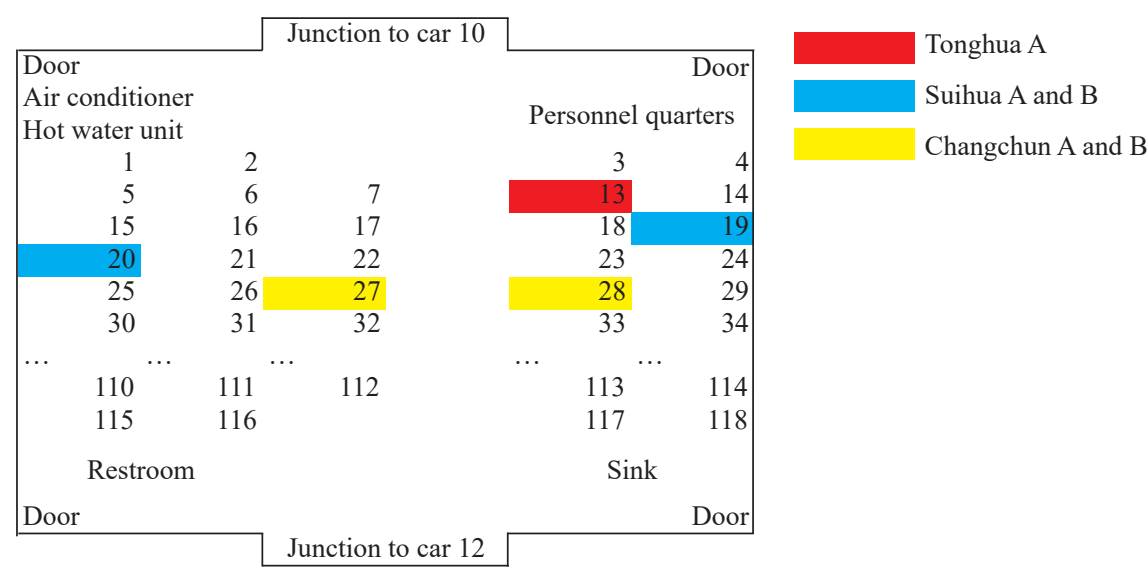

FIGURE 1. A diagram of where the patients in this outbreak were seated in Car 11 of the K350 train that departs daily from Jiamusi Station at $09: 28$ and arrives in Beijing at $13: 00$ the next day.

K350 passes through 18 stations across 4 provinces (Heilongjiang, Jilin, Liaoning, and Hebei) and 2 municipalities (Tianjin and Beijing). The total journey time is approximately 27 hours and 32 minutes.

TABLE 1. A diagram showing the overlap of patients found in this outbreak.

\begin{tabular}{|c|c|c|c|c|c|c|c|}
\hline Station \# & Station name & Arrival time & Departure time & Standby length (minutes) & Tonghua A & Suihua A\&B & Changchun A\&B \\
\hline 1 & Jiamusi & $9: 28$ & $9: 28$ & - & & & \\
\hline 2 & Nancha & $11: 22$ & $11: 25$ & 3 & & & \\
\hline 3 & Langxiang & $12: 04$ & $12: 06$ & 2 & & & \\
\hline 4 & Tieli & $13: 09$ & $13: 11$ & 2 & & & \\
\hline 5 & Suihua & $14: 33$ & $14: 41$ & 8 & & & \\
\hline 6 & Harbin West & $16: 28$ & $17: 00$ & 32 & & & \\
\hline 7 & Shuangchengbao & $17: 33$ & $17: 36$ & 3 & & & \\
\hline 8 & Dehui & $18: 44$ & $18: 46$ & 2 & & & \\
\hline 9 & Changchun & $19: 42$ & 20:05 & 23 & & & \\
\hline 10 & Gongzhuling & $20: 43$ & $20: 45$ & 2 & & & \\
\hline
\end{tabular}

Note: Patients Suihua A and B (blue) were likely the source of COVID-19 and likely transmitted the infection to Tonghua A (red) and Changchun $A$ and $B$ (yellow).

\section{DISCUSSION}

The linkage of cases in this outbreak of COVID-19 across several cities and provinces in northern China suggests that current measures must be diligently maintained and citizens must closely follow guidelines and promptly seek testing when they suspect they have symptoms. Though the K350 train is air conditioned, the cold winter conditions lead to worse ventilation and can lead to periods of extended exposure if a source of infection is close by.

As the Spring Festival (Lunar New Year) approaches, a period of extensive travel across China will commence as residents return home to their families, and existing prevention and control measures will be tested. A high potential of superspreading events and of outbreaks spanning entire regions exists, and to prevent extended periods of lockdown like that most recently occurring in Hebei Province (1), one of the strongest measures in the public health arsenal of nonpharmaceutical interventions, all stakeholders must buy in.

Current measures include the following: 1) passengers boarding from low-risk areas should provide negative nucleic acid test results within the most recent seven days; 2) medium to high-risk areas will have ticket purchases suspended, passengers will be prevented from boarding, and purchased tickets will be refunded online; 3) passengers will be given basic health precaution training including advocating strict mask wearing, proper hand hygiene, and supervision of these measures by train staff; and 4) railway operating companies will register passenger information, increase monitoring and enforcement of public health 
measures, and promptly report any abnormalities.

Despite careful measures reducing the inflow of individuals coming into China, the new variants of COVID-19 have been reported in China already including the VUI202012/01 variant first emerging in the United Kingdom (2) and the 501Y.V2 variant first emerging in South Africa (3). In addition, COVID-19 has been shown to spread via frozen food products and related packaging (4), which was not established at the beginning of the pandemic. All these events indicate the process of understanding and combatting a new virus threat, and how in order to reduce the disease burden on the population, all aspects of the response, including policymakers, implementers, and the public, must contribute equally while the measures can be developed, fine-tuned, and perfected.

Acknowledgements: Yali Wang, Ruiqi Ren, Dan Li, Yecheng Yao, Wufei Tian, Xuejia Li, and Ping Yu from China CDC; and Chunxia Jin from Hebei Institute of Plague Control.

Funding: National Natural Science Foundation (grant number 71934002).

doi: $10.46234 / \mathrm{ccdcw} 2021.026$

\# Corresponding authors: Qun Li, liqun@chinacdc.cn; Biao Huang, huangbiao2000@sohu.com.
${ }^{1}$ Public Health Emergency Center, Chinese Center for Disease Control and Prevention, Beijing, China; ${ }^{2}$ Department of Epidemiology and Biostatistics, School of Public Health, Peking University Health Science Center, Beijing, China; ${ }^{3}$ Jilin Center for Disease Control and Prevention, Changchun, Jilin, China; ${ }^{4}$ Chinese Center for Disease Control and Prevention, Beijing, China; ${ }^{5}$ School of Public Health, Weifang Medical University, Weifang, Shandong, China.

\& Joint first authors.

Submitted: January 15, 2021; Accepted: February 01, 2021

\section{REFERENCES}

1. Qi SX, Zhao X, Hao P, Liu NK, Gao GF, Song Y, et al. Two reemergent cases of COVID-19 - Hebei Province, China, January 2, 2021. China CDC Wkly 2021;3(2):25 - 7. http://dx.doi.org/10.46234/ccdcw2021. 006.

2. Chen HY, Huang XY, Zhao Z, Song Y, Hao P, Jiang H, et al. The first case of new variant COVID-19 originating in the United Kingdom detected in a returning student - Shanghai Municipality, China, December 14, 2020. China CDC Wkly 2021;3(1):1-3. http://dx.doi.org/10.46234/ccdcw2020.270.

3. Chen FJ, Li BS, Hao P, Song Y, Xu WB, Liu NK, et al. A case of new variant COVID-19 first emerging in South Africa detected in airplane pilot - Guangdong Province, China, January 6, 2021. China CDC Wkly 2021;3(2):28 - 9. http://dx.doi.org/10.46234/ccdcw2021.007.

4. Bai L, Wang YR, Wang YBN, Wu YN, Li N, Liu ZP. Controlling COVID-19 transmission due to contaminated imported frozen food and food packaging. China CDC Wkly 2021;3(2):30 - 3. http://dx.doi.org/ $10.46234 / \mathrm{ccdcw} 2021.008$. 